\title{
ON THE ACOUSTIC SCATTERING AMPLITUDE FOR A MULTI-LAYERED SCATTERER
}

\author{
CHRISTODOULOS ATHANASIADIS ${ }^{1}$
}

(Received 30 January 1996; revised 15 May 1996)

\begin{abstract}
We consider the boundary-value problems corresponding to the scattering of a timeharmonic acoustic plane wave by a multi-layered obstacle with a sound-soft, hard or penetrable core. Firstly, we construct in closed forms the normalized scattering amplitudes and prove the classical reciprocity and scattering theorems for these problems. These results are then used to study the spectrum of the scattering amplitude operator. The scattering cross-section is expressed in terms of the forward value of the corresponding normalized scattering amplitude. Finally, we develop a more general theory for scattering relations.
\end{abstract}

\section{Introduction}

This paper is concerned with the reciprocity principle and the general scattering theorem for the normalized scattering amplitude corresponding to the scattering of time-harmonic acoustic plane waves by a multi-layered scatterer. A scatterer of this type is a nested body consisting of a finite number of homogeneous layers. On the surfaces that describe this tessellation are imposed transmission conditions, that physically express the continuity of the medium and the equilibrium of the forces acting on it. In the interior of the scatterer there exists a sound-soft, hard or penetrable core.

An integrated and systematic theory for the scattering of acoustic waves was presented by Kleinman and Senior in [15]. Twersky in [19] proved reciprocity and scattering theorems for both soft and hard obstacles, and using low frequency expansions, he obtained the leading-term approximation of the real part of the scattering amplitude by direct application of the scattering theorem. Dassios developed the low-frequency theory for acoustic scattering by a soft body [9] and by a penetrable body with either a soft or a rigid core [10]. For existence and uniqueness of solutions of acoustic

\footnotetext{
${ }^{1}$ Department of Mathematics, University of Athens, Panepistemiopolis, GR 15784 Athens, Greece (C) Australian Mathematical Society, 1998, Serial-fee code 0334-2700/98
} 
scattering problems we refer to the books by Colton and Kress [6], [7] and Jones [13]. The unique-solvability of the transmission problem of acoustics was studied in [16] by Kress and Roach and in [14] by Kleinman and Martin, using the boundary integral equations method. The well-posedness of the resistive and conductive problems for the Helmholtz equation was proved in [1]. A simple proof of the normality of the far field operator is given in [8].

Reciprocity relations and general scattering theorems connecting the three normalized spherical scattering amplitudes of the elastic theory of scattering, were proved by Dassios, Kiriaki and Polyzos in [11]. Twersky in [20] proved reciprocity, scattering and optical theorems for electromagnetic waves.

Acoustic scattering by a multi-layered scatterer has been studied by Sabatier in [18]. He constructed a Green's function for the "mixed potential-impedance" equation, using the boundary integral equations method. Multi-layered scatterers were used in [2] and [12] for scattering of elecromagnetic waves. A nested scatterer with an infinite number of layers was described in [5]. In this case, using a generalized solutions approach, existence and uniqueness of solutions were proved. Multi-layered ellipsoidal scatterers with sound-soft and -hard core were used in [4] and [3], respectively, where, working in ellipsoidal geometry, the first three low-frequency coefficients were obtained.

In Section 2 we formulate the three scattering problems for which the normalized scattering amplitudes will be studied. In Section 3, using an integral representation of the scattered field, we construct the scattering amplitude for each problem. The main results of the paper, that is, reciprocity and scattering theorems are included in Section 4. Also, Herglotz wave functions are used as incident fields and the scattering amplitude operator is studied. In Section 5, we state the optical theorem, that is a connection of the scattering amplitude to the cross-section. Furthermore we discuss problems with resistive and conductive boundary conditions on the surface of the core.

Finally, in Section 6 we consider a general boundary-value problem and develop a more general theory for scattering relations.

\section{Acoustic scattering by a multi-layered scatterer}

Let $\Omega$ be a bounded and closed subset of $\mathbb{R}^{3}$ and a $C^{2}$-boundary $S_{0}$. We consider $\Omega$ to be a nested body, consisting of annuli-like regions $\Omega_{j}$, divided by means of closed and $C^{2}$-surfaces $S_{j}, j=1,2, \ldots, \mu$. Each $S_{j}$ surrounds $S_{j+1}$ and $S_{i} \cap S_{j}=\emptyset$, for any $i \neq j$. The region $\Omega_{\mu+1}$, within which lies the origin, is the core of the scatterer. The core may be a sound-soft, a -hard or a -penetrable body, that is, on the surface of the core Dirichlet, Neumann or transmission boundary conditions are satisfied, respectively. The physics of the problem is determined by the values of the mass 
density $\rho_{j}$ associated with the velocity field, and the compressibility $\gamma_{j}$ associated with the pressure field in each layer $\Omega_{j}$. The exterior of the scatterer, $\Omega_{0}$, is an infinite homogeneous isotropic medium with mass density $\rho_{0}$ and compressibility $\gamma_{0}$. There is one normal unit vector $\hat{\mathbf{n}}(\mathbf{r})$ at each point $\mathbf{r}$ of any surface $S_{j}$ pointing into $\Omega_{j}$. The wave number $k_{j}$ in $\Omega_{j}$ is a positive real number and it is expressed in terms of the wave number $k_{0}$ of the exterior space $\Omega_{0}$, as

$$
k_{j}^{2}=\frac{\gamma_{0} \rho_{j}}{\gamma_{j} \rho_{0}} k_{0}^{2}
$$

(see [4]). The set $\Omega$ described above, is a multi-layered scatterer with a core. We assume that a time-harmonic acoustic plane wave $u^{i}$ is incident upon the obstacle $\Omega$. The total acoustic field $u_{j}$ in $\Omega_{j}$, satisfies the Helmholtz equation

$$
\Delta u_{j}+k_{j}^{2} u_{j}=0 \quad \text { in } \Omega_{j} .
$$

Furthermore the total exterior field $u_{0}$ is given by

$$
u_{0}=u^{i}+u^{s} \quad \text { in } \Omega_{0},
$$

where $u^{s}$ is the scattered field satisfying the Sommerfeld radiation condition

$$
\hat{\mathbf{r}} \cdot \operatorname{grad} u^{s}(\mathbf{r})-i k_{0} u^{s}(\mathbf{r})=o\left(\frac{1}{r}\right), \quad r \rightarrow \infty
$$

uniformly in all directions $\hat{\mathbf{r}} \in S^{2}$, where $S^{2}$ is the unit sphere.

On the surface $S_{j}$ we have the transmission conditions

$$
\left.\begin{array}{l}
u_{j}=u_{j+1} \\
\frac{\partial u_{j}}{\partial n}=\frac{\rho_{j}}{\rho_{j+1}} \frac{\partial u_{j+1}}{\partial n}
\end{array}\right\} \quad \text { on } S_{j} \quad j=0,1, \ldots, \mu-1 .
$$

According to the boundary conditions on the surface of the core, we have the following three problems.

Problem $\left(P_{D}\right): \quad$ Determine $u_{j} \in C^{2}\left(\Omega_{j}\right) \cap C^{1}\left(\bar{\Omega}_{j}\right), j=0,1, \ldots, \mu$, satisfying (2) to (5) and

$$
u_{\mu}=0 \quad \text { on } S_{\mu} .
$$

Problem $\left(P_{N}\right):$ Determine $u_{j} \in C^{2}\left(\Omega_{j}\right) \cap C^{1}\left(\bar{\Omega}_{j}\right), j=0,1, \ldots, \mu$, satisfying (2) to (5) and

$$
\frac{\partial u_{\mu}}{\partial n}=0 \quad \text { on } S_{\mu}
$$


Problem $\left(P_{T}\right): \quad$ Determine $u_{j} \in C^{2}\left(\Omega_{j}\right) \cap C^{1}\left(\bar{\Omega}_{j}\right), j=0,1, \ldots, \mu+1$, satisfying (2) to (5) and

$$
\left.\begin{array}{l}
u_{\mu}=u_{\mu+1} \\
\frac{\partial u_{\mu}}{\partial n}=\frac{\rho_{\mu}}{\rho_{\mu+1}} \frac{\partial u_{\mu+1}}{\partial n}
\end{array}\right\} \quad \text { on } S_{\mu}
$$

The proof of the well-posedness of these boundary value problems, based on proving existence and uniqueness for the solution of the corresponding integral equation formulation of the problems, is discussed in [5] and [18].

Our aim on the present work is to prove the reciprocity principle and the scattering theorem for the above three problems.

\section{Construction of the scattering amplitudes}

It is well known [7] that the scattered field, as a radiating solution of the Helmholtz equation, has the integral representation

$$
u^{s}(\mathbf{r})=\int_{s_{0}}\left[u^{s}\left(\mathbf{r}^{\prime}\right) \frac{\partial \phi_{0}\left(\mathbf{r}, \mathbf{r}^{\prime}\right)}{\partial n\left(\mathbf{r}^{\prime}\right)}-\phi_{0}\left(\mathbf{r}, \mathbf{r}^{\prime}\right) \frac{\partial u^{s}\left(\mathbf{r}^{\prime}\right)}{\partial n}\right] d s\left(\mathbf{r}^{\prime}\right), \quad \mathbf{r} \in \Omega_{0}
$$

where

$$
\phi_{0}\left(\mathbf{r}, \mathbf{r}^{\prime}\right)=\frac{1}{4 \pi} \frac{e^{i k_{0}\left|\mathbf{r}-\mathbf{r}^{\prime}\right|}}{\left|\mathbf{r}-\mathbf{r}^{\prime}\right|}, \quad \mathbf{r} \neq \mathbf{r}^{\prime}
$$

is the fundamental solution of (2) in $\Omega_{0}$. Closely related to the scattered field is the normalized scattering amplitude $g$, which is defined by the relation

$$
u^{s}(\mathbf{r})=g(\hat{\mathbf{r}}) h\left(k_{0} r\right)+\mathrm{O}\left(\frac{1}{r^{2}}\right), \quad r \rightarrow \infty,
$$

where $h(x)=e^{i x} / i x$ is the zeroth-order spherical Hankel function of the first kind. In order to express $g$ in closed form we work as follows.

Inserting $u^{s}=u_{0}-u^{i}$ in (9) and taking into account that $u^{i}$ is an entire solution of the Helmholtz equation in $\Omega_{0}$ we obtain

$$
u^{s}(\mathbf{r})=\int_{s_{0}}\left[u_{0}\left(\mathbf{r}^{\prime}\right) \frac{\partial \phi_{0}\left(\mathbf{r}, \mathbf{r}^{\prime}\right)}{\partial n\left(\mathbf{r}^{\prime}\right)}-\phi_{0}\left(\mathbf{r}, \mathbf{r}^{\prime}\right) \frac{\partial u_{0}\left(\mathbf{r}^{\prime}\right)}{\partial n}\right] d s\left(\mathbf{r}^{\prime}\right), \quad \mathbf{r} \in \Omega_{0}
$$

Now, applying successively Green's first theorem on $u_{j}\left(\mathbf{r}^{\prime}\right), \phi_{0}\left(\mathbf{r}, \mathbf{r}^{\prime}\right)$ in $\Omega_{j}$ and taking into account that $u_{j}\left(\mathbf{r}^{\prime}\right), \phi_{0}\left(\mathbf{r}, \mathbf{r}^{\prime}\right)$ are solutions of (2) in $\Omega_{j}$ and $\Omega_{0}$ respectively, and introducing the transmission conditions (5) and boundary conditions $(6)$ for $\left(P_{D}\right),(7)$ for 
$\left(P_{N}\right)$ and transmission conditions $(8)$ for $\left(P_{T}\right)$, we derive the following representations of the scattered fields

$$
\begin{aligned}
& u_{D}^{S}(\mathbf{r})=V^{(\mu)}(\mathbf{r})+W^{(\mu)}(\mathbf{r})+F_{D}(\mathbf{r}), \quad \mathbf{r} \in \Omega_{0}, \\
& u_{N}^{S}(\mathbf{r})=V^{(\mu)}(\mathbf{r})+W^{(\mu)}(\mathbf{r})+F_{N}(\mathbf{r}), \quad \quad \mathbf{r} \in \Omega_{0}, \\
& u_{T}^{S}(\mathbf{r})=V^{(\mu+1)}(\mathbf{r})+W^{(\mu+1)}(\mathbf{r}), \quad \mathbf{r} \in \Omega_{0},
\end{aligned}
$$

for the problems $\left(P_{D}\right),\left(P_{N}\right)$ and $\left(P_{T}\right)$ respectively, where

$$
\begin{aligned}
V^{(m)}(\mathbf{r}) & =k_{0}^{2} \sum_{j=1}^{m}\left(\frac{\gamma_{0}}{\gamma_{j}}-1\right) \int_{\Omega_{j}} u_{j}\left(\mathbf{r}^{\prime}\right) \phi_{0}\left(\mathbf{r}, \mathbf{r}^{\prime}\right) d v\left(\mathbf{r}^{\prime}\right), \quad \mathbf{r} \in \Omega_{0}, \\
W^{(m)}(\mathbf{r}) & =\sum_{j=1}^{m}\left(1-\frac{\rho_{0}}{\rho_{j}}\right) \int_{\Omega_{j}} \operatorname{grad} u_{j}\left(\mathbf{r}^{\prime}\right) \cdot \operatorname{grad}_{\mathbf{r}} \phi_{0}\left(\mathbf{r}, \mathbf{r}^{\prime}\right) d v\left(\mathbf{r}^{\prime}\right), \quad \mathbf{r} \in \Omega_{0},
\end{aligned}
$$

for $m=\mu, \mu+1$,

$$
\begin{aligned}
& F_{D}(\mathbf{r})=-\frac{\rho_{0}}{\rho_{\mu}} \int_{S_{\mu}} \phi_{0}\left(\mathbf{r}, \mathbf{r}^{\prime}\right) \frac{\partial u_{\mu}\left(\mathbf{r}^{\prime}\right)}{\partial n} d s\left(\mathbf{r}^{\prime}\right), \quad \mathbf{r} \in \Omega_{0}, \\
& F_{N}(\mathbf{r})=\int_{S_{\mu}} u_{\mu}\left(\mathbf{r}^{\prime}\right) \frac{\partial \phi_{0}\left(\mathbf{r}, \mathbf{r}^{\prime}\right)}{\partial n\left(\mathbf{r}^{\prime}\right)} d s\left(\mathbf{r}^{\prime}\right), \quad \mathbf{r} \in \Omega_{0} .
\end{aligned}
$$

The functions $V^{(m)}(\mathbf{r})$ and $W^{(m)}(\mathbf{r})$ are dependent on the physical parameters of the body and express the contribution of the layers to the scattered field. The surface integrals (18) and (19) represent the effect of the core on the scattered field.

From the asymptotic form

$$
\left|\mathbf{r}-\mathbf{r}^{\prime}\right|=r-\hat{\mathbf{r}} \cdot \mathbf{r}+\mathrm{O}\left(\frac{1}{r}\right), \quad r \rightarrow \infty
$$

we derive [7]

$$
\begin{array}{cl}
\phi_{0}\left(\mathbf{r}, \mathbf{r}^{\prime}\right) & =\frac{i k_{0}}{4 \pi} h\left(k_{0} r\right) e^{-i k_{0} \hat{\mathbf{r}} \cdot \mathbf{r}^{\prime}}+\mathrm{O}\left(\frac{1}{r^{2}}\right), \quad r \rightarrow \infty, \\
\frac{\partial \phi_{0}\left(\mathbf{r}, \mathbf{r}^{\prime}\right)}{\partial n\left(\mathbf{r}^{\prime}\right)}=\frac{i k_{0}}{4 \pi} h\left(k_{0} r\right) \frac{\partial e^{-i k_{0} \hat{r} \cdot \mathbf{r}^{\prime}}}{\partial n\left(\mathbf{r}^{\prime}\right)}+O\left(\frac{1}{r^{2}}\right), \quad r \rightarrow \infty .
\end{array}
$$


Substituting (21) and (22) into (16)-(19) we take the asymptotic forms, for $r \rightarrow \infty$

$$
\begin{aligned}
V^{(m)}(\mathbf{r}) & =h\left(k_{0} r\right)\left[\frac{i k_{0}^{3}}{4 \pi} \sum_{j=1}^{m}\left(\frac{\gamma_{0}}{\gamma_{j}}-1\right) \int_{\Omega_{j}} u_{j}\left(\mathbf{r}^{\prime}\right) e^{-i k_{0} \hat{\mathbf{r}} \cdot \mathbf{r}^{\prime}} d v\left(\mathbf{r}^{\prime}\right)\right]+\mathrm{O}\left(\frac{1}{r^{2}}\right) \\
& \equiv h\left(k_{0} r\right) V_{\infty}^{(m)}(\hat{\mathbf{r}})+\mathrm{O}\left(\frac{1}{r^{2}}\right) \\
W^{(m)}(\mathbf{r}) & =h\left(k_{0} r\right)\left[\frac{i k_{0}}{4 \pi} \sum_{j=1}^{m}\left(1-\frac{\rho_{0}}{\rho_{j}}\right) \int_{\Omega_{j}} \operatorname{grad} u_{j}\left(\mathbf{r}^{\prime}\right) \cdot \operatorname{grad}_{\mathbf{r}^{\prime}} e^{-i k_{0} \hat{\mathbf{r}} \cdot \mathbf{r}^{\prime}} d v\left(\mathbf{r}^{\prime}\right)\right]+\mathrm{O}\left(\frac{1}{r^{2}}\right) \\
& \equiv h\left(k_{0} r\right) W_{\infty}^{(m)}(\hat{\mathbf{r}})+\mathrm{O}\left(\frac{1}{r^{2}}\right) \\
F_{D}(\mathbf{r}) & =h\left(k_{0} r\right)\left[-\frac{i k_{0} \rho_{0}}{4 \pi \rho_{\mu}} \int_{S_{\mu}} e^{-i k_{0} \hat{\mathbf{r}} \cdot \mathbf{r}^{\prime}} \frac{\partial u_{\mu}\left(\mathbf{r}^{\prime}\right)}{\partial n} d s\left(\mathbf{r}^{\prime}\right)\right]+\mathrm{O}\left(\frac{1}{r^{2}}\right) \\
& \equiv h\left(k_{0} r\right) F_{D, \infty}(\hat{\mathbf{r}})+\mathrm{O}\left(\frac{1}{r^{2}}\right) \\
F_{N}(\mathbf{r}) & =h\left(k_{0} r\right)\left[\frac{i k_{0}}{4 \pi} \int_{S_{\mu}} u_{\mu}\left(\mathbf{r}^{\prime}\right) \frac{\partial e^{-i k_{0} \hat{\mathbf{r}} \cdot \mathbf{r}^{\prime}}}{\partial n\left(\mathbf{r}^{\prime}\right)} d s\left(\mathbf{r}^{\prime}\right)\right]+\mathrm{O}\left(\frac{1}{r^{2}}\right) \\
& \equiv h\left(k_{0} r\right) F_{N, \infty}(\hat{\mathbf{r}})+\mathrm{O}\left(\frac{1}{r^{2}}\right)
\end{aligned}
$$

Consequently, for $a=D, N, T$, we have

$$
u_{a}^{s}(\mathbf{r})=h\left(k_{0} r\right) g_{a}(\hat{\mathbf{r}})+\mathrm{O}\left(\frac{1}{r^{2}}\right), \quad r \rightarrow \infty,
$$

where the fields

$$
\begin{aligned}
& g_{D}(\hat{\mathbf{r}})=V_{\infty}^{(\mu)}(\hat{\mathbf{r}})+W_{\infty}^{(\mu)}(\hat{\mathbf{r}})+F_{D, \infty}(\hat{\mathbf{r}}), \\
& g_{N}(\hat{\mathbf{r}})=V_{\infty}^{(\mu)}(\hat{\mathbf{r}})+W_{\infty}^{(\mu)}(\hat{\mathbf{r}})+F_{N, \infty}(\hat{\mathbf{r}}), \\
& g_{T}(\hat{\mathbf{r}})=V_{\infty}^{(\mu+1)}(\hat{\mathbf{r}})+W_{\infty}^{(\mu+1)}(\hat{\mathbf{r}}),
\end{aligned}
$$

are the scattering amplitudes for the problems $\left(P_{D}\right),\left(P_{N}\right)$ and $\left(P_{T}\right)$, respectively. This argument establishes the following theorem.

THEOREM 1. The normalized scattering amplitudes for the problems $\left(P_{D}\right),\left(P_{N}\right)$ and $\left(P_{T}\right)$ are the functions $g_{D}, g_{N}$ and $g_{T}$, respectively, defined on the unit sphere $S^{2}$ and given by (28), (29) and (30).

\section{Reciprocity and scattering theorems}

In what follows, for an incident time-harmonic plane wave $u^{i}(\mathbf{r} ; \hat{\mathbf{k}})=\exp \left(i k_{0} \hat{\mathbf{k}} \cdot \mathbf{r}\right)$ we will denote the total field in $\Omega_{j}$, the scattered field and the normalized scattering 
amplitude for the problem $\left(P_{a}\right), a=D, N, T$ by writing, respectively, $u_{a, j}(\mathbf{r} ; \hat{\mathbf{k}})$, $u_{a}^{s}(\mathbf{r} ; \hat{\mathbf{k}})$ and $g_{a}(\hat{\mathbf{r}} ; \hat{\mathbf{k}})$ indicating the dependence on the incident direction $\hat{\mathbf{k}}$. Also in the sequel we shall make use Twersky's notation [19]

$$
\{u, U\}_{s}=\int_{s}\left[u(\mathbf{r}) \frac{\partial U(\mathbf{r})}{\partial n}-U(\mathbf{r}) \frac{\partial u(\mathbf{r})}{\partial n}\right] d s .
$$

We consider two incident plane waves $u^{i}(\mathbf{r} ; \hat{\mathbf{p}})=\exp \left(i k_{0} \hat{\mathbf{p}} \cdot \mathbf{r}\right)$ and $u^{i}(\mathbf{r} ; \hat{\mathbf{q}})=$ $\exp \left(i k_{0} \hat{\mathbf{q}} \cdot \mathbf{r}\right)$, propagating in the directions $\hat{\mathbf{p}}$ and $\hat{\mathbf{q}}$ respectively. Then we may state and prove the following three lemmas.

LEMMA 1. For the scattering problem $\left(P_{a}\right), a=D, N, T$ we have

$$
\left\{u_{a, 0}(\cdot ; \hat{\mathbf{p}}), u_{a, 0}(\cdot ; \hat{\mathbf{q}})\right\}_{S_{0}}=0,
$$

for all $\hat{\mathbf{p}}, \hat{\mathbf{q}} \in S^{2}$.

PROOF. Applying successively Green's second theorem on $u_{a, j}(\mathbf{r} ; \hat{\mathbf{p}})$ and $u_{a, j}(\mathbf{r} ; \hat{\mathbf{q}})$ in $\Omega_{j}$, using the transmission conditions (5) and taking into account that

$$
\int_{\Omega_{j}}\left[u_{a, j}(\mathbf{r} ; \hat{\mathbf{p}}) \Delta u_{a, j}(\mathbf{r} ; \hat{\mathbf{q}})-u_{a, j}(\mathbf{r} ; \hat{\mathbf{q}}) \Delta u_{a, j}(\mathbf{r} ; \hat{\mathbf{p}})\right] d v(\mathbf{r})=0, \quad j=1,2, \ldots, \mu+1,
$$

since both $u_{a, j}(\mathbf{r} ; \hat{\mathbf{p}})$ and $u_{a, j}(\mathbf{r} ; \hat{\mathbf{q}})$ satisfy (3), we conclude that

$$
\left\{u_{a, 0}(\cdot ; \hat{\mathbf{p}}), u_{a, 0}(\cdot ; \hat{\mathbf{q}})\right\}_{S_{0}}=\frac{\rho_{0}}{\rho_{\mu}}\left\{u_{a, \mu}(\cdot ; \hat{\mathbf{p}}), u_{a, \mu}(\cdot ; \hat{\mathbf{q}})\right\}_{S_{\mu}} .
$$

For $a=D, N$ the lemma is proved by introducing the boundary conditions (6) and (7), respectively, into (34). Inserting the transmission conditions (8) in (34), applying again Green's second theorem in $\Omega_{\mu+1}$ and using the relation (33) for $j=\mu+1$ we prove the lemma for $a=T$.

LEMMA 2. For the scattering problem $\left(P_{a}\right), a=D, N, T$ we have

$$
\left\{u^{i}(\cdot ; \hat{\mathbf{p}}), u_{a}^{s}(\cdot ; \hat{\mathbf{q}})\right\}_{S_{0}}=\left\{u^{i}(\cdot ; \hat{\mathbf{q}}), u_{a}^{s}(\cdot ; \hat{\mathbf{p}})\right\}_{S_{0}},
$$

for all $\hat{\mathbf{p}}, \hat{\mathbf{q}} \in S^{2}$.

Proof. From (3), the bilinearity of $\{\cdot, \cdot\}_{S_{0}}$ and using Lemma 1 we take

$$
\begin{aligned}
& \left\{u_{a}^{s}(\cdot ; \hat{\mathbf{p}}), u_{a}^{s}(\cdot ; \hat{\mathbf{q}})\right\}_{S_{0}}+\left\{u_{a}^{s}(\cdot ; \hat{\mathbf{p}}), u^{i}(\cdot ; \hat{\mathbf{q}})\right\}_{S_{0}} \\
& \quad+\left\{u^{i}(\cdot ; \hat{\mathbf{p}}), u_{a}^{s}(\cdot ; \hat{\mathbf{q}})\right\}_{S_{0}}+\left\{u^{i}(\cdot ; \hat{\mathbf{p}}), u^{i}(\cdot ; \hat{\mathbf{q}})\right\}_{S_{0}}=0 .
\end{aligned}
$$


In view of Green's second theorem, the Helmholtz equation for the incident and the scattered wave and the radiation condition for the scattered wave we have

$$
\begin{array}{r}
\left\{u^{i}(\cdot ; \hat{\mathbf{p}}), u^{i}(\cdot ; \hat{\mathbf{q}})\right\}_{S_{0}}=0, \\
\left\{u_{a}^{s}(\cdot ; \hat{\mathbf{p}}), u_{a}^{s}(\cdot ; \hat{\mathbf{q}})\right\}_{S_{0}}=0 .
\end{array}
$$

Therefore, (36), (37) and (38) yield (35).

Furthermore we prove the following lemma, where $\bar{U}$ denotes the complex conjugate of $U$.

LEMMA 3. For the scattering problem $\left(P_{a}\right), a=D, N, T$ we have

$$
g_{a}(\hat{\mathbf{p}} ; \hat{\mathbf{q}})=\frac{i k_{0}}{4 \pi}\left\{u_{a}^{s}(\cdot ; \hat{\mathbf{q}}), \overline{u^{i}(\cdot ; \hat{\mathbf{p}})}\right\}_{S_{0}},
$$

for all $\hat{\mathbf{p}}, \hat{\mathbf{q}} \in S^{2}$.

PROOF. Let $u_{a}^{s}(\mathbf{p} ; \hat{\mathbf{q}}), a=D, N, T$ be the scattered field corresponding to the incident wave $u^{i}(\mathbf{p} ; \hat{\mathbf{q}})=\exp \left(i k_{0} \mathbf{p} \cdot \hat{\mathbf{q}}\right)$. Then, as in Theorem 1 , we use the integral representation

$$
u_{a}^{s}(\mathbf{p} ; \hat{\mathbf{q}})=\left\{u_{a}^{s}(\cdot ; \hat{\mathbf{q}}), \phi_{0}(\mathbf{p}, \cdot)\right\}_{s_{0}}, \quad \mathbf{p} \in \Omega_{0}
$$

Letting $p \rightarrow \infty$ and using the asymptotic forms (21) and (22) we conclude that

$$
u_{a}^{s}(\mathbf{p} ; \hat{\mathbf{q}})=\frac{i k_{0}}{4 \pi} h\left(k_{0} p\right)\left\{u_{a}^{s}(\cdot ; \hat{\mathbf{q}}), \overline{u^{i}(\cdot, \hat{\mathbf{p}})}\right\}_{S_{0}}+\mathrm{O}\left(\frac{1}{p^{2}}\right), \quad p \rightarrow \infty .
$$

The formula (39) comes out of direct comparison of (41) with (27).

We are now in a position to prove the reciprocity principle.

THEOREM 2. The normalized scattering amplitude $g_{a}$ of the problem $\left(P_{a}\right), a=$ $D, N, T$ satisfies the reciprocity relation

$$
g_{a}(\hat{\mathbf{p}} ; \hat{\mathbf{q}})=g_{a}(-\hat{\mathbf{q}} ;-\hat{\mathbf{p}})
$$

for all $\hat{\mathbf{p}}, \hat{\mathbf{q}} \in S^{2}$. 
PROOF. By Lemmas 2 and 3 we have

$$
\begin{aligned}
g_{a}(\hat{\mathbf{p}} ; \hat{\mathbf{q}}) & =-\frac{i k_{0}}{4 \pi}\left\{\overline{u^{i}(\cdot ; \hat{\mathbf{p}})}, u_{a}^{s}(\cdot ; \hat{\mathbf{q}})\right\}_{S_{0}} \\
& =-\frac{i k_{0}}{4 \pi}\left\{u^{i}(\cdot ;-\hat{\mathbf{p}}), u_{a}^{s}(\cdot ; \hat{\mathbf{q}})\right\}_{S_{0}} \\
& =-\frac{i k_{0}}{4 \pi}\left\{u^{i}(\cdot ; \hat{\mathbf{q}}), u_{a}^{s}(\cdot ;-\hat{\mathbf{p}})\right\}_{S_{0}} \\
& =-\frac{i k_{0}}{4 \pi}\left\{\overline{u^{i}(\cdot ;-\hat{\mathbf{q}})}, u_{a}^{s}(\cdot ;-\hat{\mathbf{p}})\right\}_{S_{0}}=g_{a}(-\hat{\mathbf{q}} ;-\hat{\mathbf{p}}),
\end{aligned}
$$

which proves the theorem.

We shall now prove the basic scattering theorem, for the above three problems.

THEOREM 3. The normalized scattering amplitude $g_{a}$ of the problem $\left(P_{a}\right), a=$ $D, N, T$ satisfies the relation

$$
g_{a}(\hat{\mathbf{p}} ; \hat{\mathbf{q}})+g_{a} \overline{(\hat{\mathbf{q}} ; \hat{\mathbf{p}})}=-\frac{1}{2 \pi} \int_{S^{2}} g_{a}(\hat{\mathbf{r}} ; \hat{\mathbf{q}}) \overline{g_{a}(\hat{\mathbf{r}} ; \hat{\mathbf{q}})} d s(\hat{\mathbf{r}}),
$$

for all $\hat{\mathbf{p}}, \hat{\mathbf{q}} \in S^{2}$.

PROOF. Let $u^{i}(\cdot ; \hat{\mathbf{q}})$ and $u^{i}(\cdot ; \hat{\mathbf{p}})$ be incident waves with incident directions $\hat{\mathbf{q}}$ and $\hat{\mathbf{p}}$, respectively. Let also $u_{a}^{s}(\cdot ; \hat{\mathbf{q}})$ and $u_{a}^{s}(\cdot ; \hat{\mathbf{p}})$ be the corresponding scattered fields for the problem $\left(P_{a}\right), a=D, N, T$. Then by the bilinearity of the form $\{\cdot, \cdot\}_{s_{0}}$ and the relation (3), we derive

$$
\begin{aligned}
\left\{\overline{u_{a, 0}(\cdot ; \hat{\mathbf{p}})}, u_{a, 0}(\cdot ; \hat{\mathbf{q}})\right\}_{S_{0}}= & \left\{\overline{u^{i}(\cdot ; \hat{\mathbf{p}})}, u^{j}(\cdot ; \hat{\mathbf{q}})\right\}_{S_{0}}+\left\{\overline{u^{i}(\cdot ; \hat{\mathbf{p}})}, u_{a}^{s}(\cdot ; \hat{\mathbf{q}})\right\}_{S_{0}} \\
& +\left\{\overline{u_{a}^{s}(\cdot ; \hat{\mathbf{p}})}, u^{i}(\cdot ; \hat{\mathbf{q}})\right\}_{S_{0}}+\left\{\overline{u_{a}^{s}(\cdot ; \hat{\mathbf{p}})}, u_{a}^{s}(\cdot ; \hat{\mathbf{q}})\right\}_{S_{0}},
\end{aligned}
$$

where $u_{a, 0}$ denotes the total field in $\Omega_{0}$ for the problem $\left(P_{a}\right)$.

By Lemma 1 we have

$$
\left\{\overline{u_{a, 0}(\cdot ; \hat{\mathbf{p}})}, u_{a, 0}(\cdot ; \hat{\mathbf{q}})\right\}_{S_{0}}=0
$$

and from (37)

$$
\left\{\overline{u^{i}(\cdot ; \hat{\mathbf{p}})}, u^{i}(\cdot ; \hat{\mathbf{q}})\right\}_{S_{0}}=0
$$


Also, from Lemma 3 it follows that

$$
\begin{aligned}
& \left\{\overline{u^{i}(\cdot ; \hat{\mathbf{p}})}, u_{a}^{s}(\cdot ; \hat{\mathbf{q}})\right\}_{S_{0}}=-\frac{4 \pi}{i k_{0}} g_{a}(\hat{\mathbf{p}} ; \hat{\mathbf{q}}), \\
& \left\{\overline{u_{a}^{s}(\cdot ; \hat{\mathbf{p}})}, u^{i}(\cdot ; \hat{\mathbf{q}})\right\}_{S_{0}}=-\frac{4 \pi}{i k_{0}} \overline{g_{a}(\hat{\mathbf{q}} ; \hat{\mathbf{p}})}
\end{aligned}
$$

Now we consider a sphere $S_{R}$, centred at the origin, with radius $R$ large enough to include the scatterer in its interior. Applying Green's second theorem on $\overline{u_{a}^{s}(\cdot ; \hat{\mathbf{p}})}$ and $u_{a}^{s}(\cdot ; \hat{\mathbf{q}})$ in the region exterior to $S_{0}$ and interior to $S_{R}$, in view of regularity of $u_{a}^{s}$ we take the relation

$$
\left\{\overline{u_{a}^{s}(\cdot ; \hat{\mathbf{p}})}, u_{a}^{s}(\cdot ; \hat{\mathbf{q}})\right\}_{S_{0}}=\left\{\overline{u_{a}^{s}(\cdot ; \hat{\mathbf{p}})}, u_{a}^{s}(\cdot ; \hat{\mathbf{q}})\right\}_{S_{\mathrm{R}}} .
$$

Letting $R \rightarrow \infty$, we pass to the radiation zone and thus we can use the asymptotic form (27) for the scattered fields. So we have

$$
\begin{aligned}
\left\{\overline{u_{a}^{s}(\cdot ; \hat{\mathbf{p}})}, u_{a}^{s}(\cdot ; \hat{\mathbf{q}})\right\}_{S_{0}} & =2 i k_{0} \int_{S_{\infty}}\left|h\left(k_{0} r\right)\right|^{2} g_{a}(\hat{\mathbf{r}} ; \hat{\mathbf{q}}) \overline{g_{a}(\hat{\mathbf{r}} ; \hat{\mathbf{p}})} d s(\mathbf{r}) \\
& =\frac{2 i}{k_{0}} \int_{S^{2}} g_{a}(\hat{\mathbf{r}} ; \hat{\mathbf{q}}) \overline{g_{a}(\hat{\mathbf{r}} ; \hat{\mathbf{p}})} d s(\hat{\mathbf{r}}) .
\end{aligned}
$$

Substituting (46)-(49) and (51) into (45), relation (44) is derived and the scattering theorem is proved.

We now assume that the incident field is a Herglotz wave function $U_{h}^{i}$, that is, an entire solution of the Helmholtz equation $\Delta u+k_{0}^{2} u=0$ of the form

$$
U_{h}^{i}(\mathbf{r})=\int_{S^{2}} h(\hat{\mathbf{q}}) e^{i k_{0} \mathbf{r} \cdot \hat{\mathbf{q}}} d s(\hat{\mathbf{q}})
$$

where $h \in L^{2}\left(S^{2}\right)$ is the kernel of $U_{h}^{i}$. If $u_{a, h}^{s}, u_{a, \varphi}^{s}$ are the scattered fields and $g_{a, h}$, $g_{a, \varphi}$ are the normalized scattering amplitudes corresponding to the problem $\left(P_{a}\right)$, $a=D, N, T$ with incident waves $U_{h}^{i}, U_{\varphi}^{i}$, respectively, then we have the following relations.

COROLLARY 1. For the problem $\left(P_{a}\right), a=D, N, T$ we have

$$
\begin{aligned}
\left\{u_{a, \varphi}^{s}, \overline{U_{h}^{i}}\right\}_{S_{0}} & =\frac{4 \pi}{i k_{0}} \int_{S^{2}} g_{a, \varphi}(\hat{\mathbf{r}}) \overline{h(\hat{\mathbf{r}})} d s(\hat{\mathbf{r}}), \\
\left\{\overline{u_{a, \varphi}^{s}}, u_{a, h}^{s}\right\}_{S_{0}} & =\frac{2 i}{k_{0}} \int_{S^{2}} \overline{g_{a, h}(\hat{\mathbf{r}})} g_{a, h}(\hat{\mathbf{r}}) d s(\hat{\mathbf{r}}) .
\end{aligned}
$$


ProOF. Applying (39) for $g_{a, \varphi}$, multiplying by $\overline{h(\hat{\mathbf{r}})}$ and integrating on $S^{2}$ we get (53). The relation (54) is a simple consequence of (51).

Let us now define the scattering amplitude operator $G_{a}: L^{2}\left(S^{2}\right) \rightarrow L^{2}\left(S^{2}\right)$, $a=D, N, T$ corresponding to the scattering amplitude $g_{a}(\hat{\mathbf{r}} ; \hat{\mathbf{q}})$ by

$$
\left(G_{a} h\right)(\hat{\mathbf{r}})=\int_{S^{2}} g_{a}(\hat{\mathbf{r}} ; \hat{\mathbf{q}}) h(\hat{\mathbf{q}}) d s(\hat{\mathbf{q}}) .
$$

We note that $G_{a} h$ is the scattering amplitude of the problem $\left(P_{a}\right)$ corresponding to the incident wave $U_{h}^{i}$, [7].

We assume that the incident fields in Theorem 3 are the Herglotz wave functions $U_{h}^{i}$ and $U_{\varphi}^{i}$. Then, using the inner product on $L^{2}\left(S^{2}\right),(\cdot, \cdot)$, and taking into account the relations (53), (54), the basic scattering theorem takes the following form [8].

COROLLARY 2. The scattering amplitude operator $G_{a}$ corresponding to the problem $\left(P_{a}\right), a=D, N, T$ satisfies the relation

$$
\left(G_{a} h, \varphi\right)+\left(h, G_{a} \varphi\right)=-\frac{1}{2 \pi}\left(G_{a} h, G_{a} \varphi\right)
$$

We shall now conclude this section by proving some properties of $G_{a}$.

COROLLARY 3. The scattering amplitude operator $G_{a}, a=D, N, T$ has a countable number of eigenvalues. All the eigenvalues $\lambda \in \mathbb{C}$ lie on the circle $|\lambda|^{2}+4 \pi \operatorname{Re} \lambda=0$.

PROOF. From Corollary 2 we have

$$
\left(h, G_{a}^{*} \varphi\right)+\left(h, G_{a} \varphi\right)=-\frac{1}{2 \pi}\left(h, G_{a}^{*} G_{a} \varphi\right),
$$

for all $h, \varphi \in L^{2}\left(S^{2}\right)$, where $G_{a}^{*}$ is the $L^{2}$ adjoint of $G_{a}$, given by

$$
\left(G_{a}^{*} h\right)(\hat{\mathbf{r}})=\int_{S^{2}} \overline{g_{a}(\hat{\mathbf{p}} ; \hat{\mathbf{r}})} h(\hat{\mathbf{p}}) d s(\hat{\mathbf{p}})
$$

The relation (57) implies that

$$
G_{a}^{*}+G_{a}=-\frac{1}{2 \pi} G_{a}^{*} G_{a}
$$

Using the reciprocity Theorem 2 we take, $[7$, p. 57],

$$
\left(G_{a}^{*} h\right)(\hat{\mathbf{r}})=\overline{\left(G_{a} \varphi\right)(-\hat{\mathbf{r}})}
$$


where $\varphi(\hat{\mathbf{p}})=\overline{h(-\hat{\mathbf{p}})}$. So we can conclude that

$$
G_{a}^{*}+G_{a}=-\frac{1}{2 \pi} G_{a} G_{a}^{*}
$$

Consequently

$$
G_{a} G_{a}^{*}=G_{a}^{*} G_{a},
$$

that is, $G_{a}$ is normal and hence has a countable number of eigenvalues.

Let $G_{a} h=\lambda h, \lambda \in \mathbb{C}$. Then, applying Corollary 2 for $h=\varphi \neq 0$ we take

$$
|\lambda|^{2}+4 \pi \operatorname{Re} \lambda=0 .
$$

\section{The scattering cross-section}

The scattering cross-section is defined as the ratio of the time average rate (over a period) at which energy is scattered by the multi-layered scatterer, to the corresponding time average rate at which the energy of the incident wave crosses a unit area normal to the direction of propagation. The scattering cross-section has the dimensions of area and is a measure of the disturbance caused by the multi-layered scatterer to the incident wave. It is proved, as in the case of a single scatterer [19], that the scattering cross-section is connected to the scattering amplitude via the relation

$$
\sigma_{a}=\frac{1}{k_{0}^{2}} \int_{S^{2}}\left|g_{a}(\hat{\mathbf{r}} ; \hat{\mathbf{p}})\right|^{2} d s(\hat{\mathbf{r}}),
$$

for $a=D, N, T$.

When the multi-layered scatterer has inversion symmetry, that is, when the scattering amplitude in invariant under an interchange of the direction of observation $\hat{\mathbf{p}}$ and the direction of incidence $\hat{\mathbf{q}}$, then the reciprocity and scattering theorems reduce to

$$
\begin{gathered}
g_{a}(\hat{\mathbf{p}} ; \hat{\mathbf{q}})=g_{a}(\hat{\mathbf{q}} ; \hat{\mathbf{p}}), \\
\operatorname{Re} g_{a}(\hat{\mathbf{p}} ; \hat{\mathbf{q}})=-\frac{1}{4 \pi} \int_{S^{2}} g_{a}(\hat{\mathbf{r}} ; \hat{\mathbf{q}}) \overline{g_{a}(\hat{\mathbf{r}} ; \hat{\mathbf{p}})} d s(\hat{\mathbf{r}}),
\end{gathered}
$$

respectively. Applying the relation (65) for $\hat{\mathbf{p}}=\hat{\mathbf{q}}$ and taking into account (63) we conclude that

$$
\sigma_{a}=-\frac{4 \pi}{k_{0}^{2}} \operatorname{Re} g_{a}(\hat{\mathbf{p}} ; \hat{\mathbf{p}}) .
$$

Formula (66) is the acoustic version of the well-known [20] optical theorem of electromagnetic waves. 
REMARK 1. When $\mu=1$, the multi-layered scatterer degenerates to a penetrable scatterer with a core for which acoustic low-frequency theory was developed in [10]. On the other hand when $\rho_{0}=\rho_{1}=\ldots=\rho_{\mu} \neq \rho_{\mu+1}$ and $\gamma_{0}=\gamma_{1}=\ldots=\gamma_{\mu} \neq \gamma_{\mu+1}$, then the layers disappear and the multi-layered scatterer coincides with its core. In this case problems $\left(P_{D}\right),\left(P_{N}\right)$ and $\left(P_{T}\right)$ reduce to Dirichlet, Neumann and transmission boundary value problems, respectively. Hence reciprocity, scattering and optical theorems for sound - soft [9], - hard [19] and penetrable [9] scatterers follow from the corresponding results for the multi-layered scatterer. When $\rho_{0} \neq \rho_{1}=\rho_{2}=\ldots=$ $\rho_{\mu+1}$ and $\gamma_{0} \neq \gamma_{1}=\gamma_{2}=\ldots=\gamma_{\mu+1}$, then scattering occurs on the surface $S_{0}$ only, and $\left(P_{a}\right)$ becomes a transmission problem for a single scatterer.

REMARK 2. More general boundary conditions can be imposed on the surface of the core. We consider either resistive-transmission conditions

$$
\left.\begin{array}{l}
u_{\mu}=u_{\mu+1}, \\
\frac{\partial u_{\mu}}{\partial n}=\frac{\rho_{\mu}}{\rho_{\mu+1}} \frac{\partial u_{\mu+1}}{\partial n}-\frac{i k_{\mu}}{\eta} u_{\mu}
\end{array}\right\} \quad \text { on } S_{\mu}
$$

or conductive transmission conditions

$$
\left.\begin{array}{l}
u_{\mu}=u_{\mu+1}+\frac{i \eta}{\rho_{\mu} k_{\mu}} \frac{\partial u_{\mu}}{\partial \eta}, \\
\frac{\partial u_{\mu}}{\partial n}=\frac{\rho_{\mu}}{\rho_{\mu+1}} \frac{\partial u_{\mu+1}}{\partial n}
\end{array}\right\} \quad \text { on } S_{\mu}
$$

where the function $\eta$ is Hölder continuous on $S_{\mu}$ [1]. In this case, the construction of the normalized scattering amplitude is done as in Theorem 1 and the basic Lemma 2 remains valid. Thus, all the previous results, that is, reciprocity, scattering and optical theorems, hold for the problems corresponding to these boundary conditions.

\section{A more general theory}

From (34) it follows that the effect of the layers on the solutions of the scattering problems $\left(P_{a}\right), a=D, N, T$ is transferred on the core. Hence the derivation of the previous scattering relations is largely independent of the composition of the obstacle. This suggests to us to develop a more general theory for scattering relations, using a more general boundary condition on $S_{0}$, omitting the layers.

Let $\Lambda$ be a linear operator acting on a space of functions defined on $S_{0}$. We denote by $\Lambda^{*}$ the adjoint of $\Lambda$ with respect to the pairing

$$
\langle\varphi, \psi\rangle:=\int_{S_{0}} \varphi \psi d s
$$


We introduce the linear space $F\left(\Omega_{0}\right)$ of all complex-valued functions $u \in C^{2}\left(\Omega_{0}\right) \cap$ $C^{1}\left(\overline{\Omega_{0}}\right)$ for which $\Lambda u$ on $S_{0}$ is defined. We consider the following boundary value problem $\left(P_{\Lambda}\right)$.

Determine a function $u \in F\left(\Omega_{0}\right)$ which satisfies

$$
\begin{array}{rr}
\Delta u+k^{2} u=0 & \text { in } \Omega_{0}, \\
\frac{\partial u}{\partial n}=\Lambda u & \text { on } S_{0}, \\
u=u^{s}+u^{i} & \text { in } \Omega_{0},
\end{array}
$$

where $u^{i}$ is an incident wave and $u^{s}$ is the scattered field satisfying the Sommerfeld radiation condition. We assume that the problem $\left(P_{\Lambda}\right)$ has a unique solution for each given incident wave. For the solvability of boundary value problems of this type we refer to [17]. We define the operator $A$ on $F\left(\Omega_{0}\right) \times F\left(\Omega_{0}\right)$ by

$$
A(\varphi, \psi):=\langle\varphi, \Lambda \psi\rangle-\langle\Lambda \varphi, \psi\rangle
$$

As in Section 4, for an incident wave $u^{i}(\mathbf{r} ; \hat{\mathbf{p}})=\exp (i k \hat{\mathbf{p}} \cdot \mathbf{r})$ we will denote the total field in $\Omega_{0}$ and the scattering field for the problem $\left(P_{\Lambda}\right)$ by writing, respectively, $u(\mathbf{r} ; \hat{\mathbf{p}})$ and $u^{s}(\mathbf{r} ; \hat{\mathbf{p}})$.

LEMMA 4. For the boundary value problem $\left(P_{\Lambda}\right)$ we have

$$
\begin{gathered}
\{u(\cdot ; \hat{\mathbf{p}}), u(\cdot ; \hat{\mathbf{q}})\}_{S_{0}}=A(u(\cdot ; \hat{\mathbf{p}}), u(\cdot ; \hat{\mathbf{q}})), \\
\left\{u^{i}(\cdot ; \hat{\mathbf{p}}), u^{s}(\cdot ; \hat{\mathbf{q}})\right\}_{S_{0}}+\left\{u^{s}(\cdot ; \hat{\mathbf{p}}), u^{i}(\cdot ; \hat{\mathbf{q}})\right\}_{S_{0}}=A(u(\cdot ; \hat{\mathbf{p}}), u(\cdot ; \hat{\mathbf{q}})),
\end{gathered}
$$

for all $\hat{\mathbf{p}}, \hat{\mathbf{q}} \in S^{2}$.

PROOF. The proof of (74) follows immediately if we make use of the boundary condition (71). From (72) and the bilinearity of the form $\{\cdot ; \cdot\}_{S_{0}}$ we have

$$
\begin{aligned}
\{u(\cdot ; \hat{\mathbf{p}}), u(\cdot ; \hat{\mathbf{q}})\}_{S_{0}}= & \left\{u^{s}(\cdot ; \hat{\mathbf{p}}), u^{s}(\cdot ; \hat{\mathbf{q}})\right\}_{S_{0}}+\left\{u^{s}(\cdot ; \hat{\mathbf{p}}), u^{i}(\cdot ; \hat{\mathbf{q}})\right\}_{S_{0}} \\
& +\left\{u^{i}(\cdot ; \hat{\mathbf{p}}), u^{s}(\cdot ; \hat{\mathbf{q}})\right\}_{S_{0}}+\left\{u^{i}(\cdot ; \hat{\mathbf{p}}), u^{i}(\cdot ; \hat{\mathbf{q}})\right\}_{S_{0}}
\end{aligned}
$$

Taking into account, as in the proof of Lemma 2, that

$$
\left\{u^{s}(\cdot ; \hat{\mathbf{p}}), u^{s}(\cdot ; \hat{\mathbf{q}})\right\}_{S_{0}}=\left\{u^{i}(\cdot ; \hat{\mathbf{p}}), u^{i}(\cdot ; \hat{\mathbf{q}})\right\}_{S_{0}}=0
$$

and using (74), we have (75). 
If a time-harmonic plane wave $\boldsymbol{u}^{i}(\mathbf{p} ; \hat{\mathbf{q}})=\exp (i k \hat{\mathbf{q}} \cdot \mathbf{p})$ is incident upon the obstacle $\Omega$, the normalized scattering amplitude $g$ is defined, as in (11), by the relation

$$
u^{s}(\mathbf{p} ; \hat{\mathbf{q}})=g(\hat{\mathbf{p}} ; \hat{\mathbf{q}}) h(k p)+\mathrm{O}\left(\frac{1}{p^{2}}\right), \quad p \rightarrow \infty .
$$

Using the fundamental solution of the Helmholtz equation (70) and working as in Lemma 3 we conclude that

$$
g(\hat{\mathbf{p}} ; \hat{\mathbf{q}})=\frac{i k}{4 \pi}\left\{u^{s}(\cdot ; \hat{\mathbf{q}}), \overline{u^{i}(\cdot ; \hat{\mathbf{p}})}\right\}_{S_{0}} .
$$

We can now prove a general reciprocity principle.

THEOREM 4. The normalized scattering amplitude of the problem $\left(P_{\Lambda}\right)$ satisfies the relation

$$
g(\hat{\mathbf{p}} ; \hat{\mathbf{q}})=g(-\hat{\mathbf{q}} ;-\hat{\mathbf{p}})+\frac{i k}{4 \pi} A(u(\cdot ; \hat{\mathbf{q}}), u(\cdot ;-\hat{\mathbf{p}})),
$$

for all $\hat{\mathbf{p}}, \hat{\mathbf{q}} \in S^{2}$.

PROOF. Using (75) and (79) we take

$$
\begin{aligned}
g(\hat{\mathbf{p}} ; \hat{\mathbf{q}}) & =\frac{i k}{4 \pi}\left\{u^{s}(\cdot ; \hat{\mathbf{q}}), u^{i}(\cdot ;-\hat{\mathbf{p}})\right\}_{s_{0}} \\
& =\frac{i k}{4 \pi}\left\{u^{s}(\cdot ; \hat{\mathbf{p}}), u^{i}(\cdot ; \hat{\mathbf{q}})\right\}_{s_{0}}+\frac{i k}{4 \pi} A(u(\cdot ; \hat{\mathbf{q}}), u(\cdot ;-\hat{\mathbf{p}})) \\
& =\frac{i k}{4 \pi}\left\{u^{s}(\cdot ;-\hat{\mathbf{p}}), \overline{u^{i}(\cdot ;-\hat{\mathbf{q}})}\right\}_{s_{0}}+\frac{i k}{4 \pi} A(u(\cdot ; \hat{\mathbf{q}}), u(\cdot ;-\hat{\mathbf{p}})) \\
& =g(-\hat{\mathbf{q}} ;-\hat{\mathbf{p}})+\frac{i k}{4 \pi} A(u(\cdot ; \hat{\mathbf{q}}), u(\cdot ;-\hat{\mathbf{p}})),
\end{aligned}
$$

which proves the theorem.

The basic scattering theorem for the problem $\left(P_{\Lambda}\right)$ has the following general form.

THEOREM 5. The normalized scattering amplitude of the problem $\left(P_{\Lambda}\right)$ satisfies the relation

$$
\begin{aligned}
g(\hat{\mathbf{p}} ; \hat{\mathbf{q}})+\overline{g(\hat{\mathbf{q}} ; \hat{\mathbf{p}})}= & -\frac{1}{2 \pi} \int_{S_{2}} g(\hat{\mathbf{r}} ; \hat{\mathbf{q}}) \overline{g(\hat{\mathbf{r}} ; \hat{\mathbf{p}})} d s(\hat{\mathbf{r}}) \\
& -\frac{i k}{4 \pi} A(\overline{u(\cdot ; \hat{\mathbf{p}})}, u(\cdot ; \hat{\mathbf{q}}))
\end{aligned}
$$

for all $\hat{\mathbf{p}}, \hat{\mathbf{q}} \in S^{2}$. 
Proof. We use the same procedure as in Theorem 3. For the problem $\left(P_{\Lambda}\right)$ equations (45), (47)-(49) and (51) remain valid and (46), in view of (74) is replaced by

$$
\{\overline{u(\cdot ; \hat{\mathbf{p}})}, u(\cdot ; \hat{\mathbf{q}})\}_{S_{0}}=A(\overline{u(\cdot ; \hat{\mathbf{p}})}, u(\cdot ; \hat{\mathbf{q}})) .
$$

Hence the theorem is proved.

We now assume that the incident fields are Herglotz wave functions. Let $u_{h}, u_{\varphi}$ be the total fields in $\Omega_{0}, u_{h}^{s}, u_{\varphi}^{s}$ the scattered fields and $g_{h}, g_{\varphi}$ the normalized scattering amplitudes corresponding to the problem $\left(P_{\Lambda}\right)$ with incident waves, the Herglotz, functions $U_{h}^{i}, U_{\varphi}^{i}$, respectively. Then, as in Corollary 1 , from (79) and (51) which remains valid for $\left(P_{\Lambda}\right)$, we have

$$
\begin{aligned}
\left\{u_{\varphi}^{s}, \overline{U_{h}^{i}}\right\}_{S_{0}} & =\frac{4 \pi}{i k} \int_{S^{2}} g_{\varphi}(\hat{\mathbf{r}}) \overline{h(\hat{\mathbf{r}})} d s(\hat{\mathbf{r}}), \\
\left\{\overline{u_{\varphi}^{s}}, u_{h}^{s}\right\}_{S_{0}} & =\frac{2 i}{k} \int_{S^{2}} \overline{g_{\varphi}(\hat{\mathbf{r}})} g_{h}(\hat{\mathbf{r}}) d s(\hat{\mathbf{r}}) .
\end{aligned}
$$

Moreover employing the scattering amplitude operator $G$, given by (55), and using (83), (84), the relation (81) becomes

$$
(G h, \varphi)+(h, G \varphi)=-\frac{1}{2 \pi}(G h, G \varphi)-\frac{i k}{4 \pi} A\left(\overline{u_{\varphi}}, u_{h}\right) .
$$

Let us now consider the case where the operator $\Lambda$ is self-adjoint. Then, for all $\varphi, \psi \in F(\Omega)$ we have

$$
\langle\varphi, \Lambda \psi\rangle=\left\langle\Lambda^{*} \varphi, \psi\right\rangle=\langle\Lambda \varphi, \psi\rangle
$$

Clearly, the following is true.

LEMMA 5. If $\Lambda$ is a self-adjoint linear operator, then

$$
A(\varphi, \psi)=0
$$

for all $\varphi, \psi \in F(\Omega)$.

Therefore, if we apply (87) in Lemma 4 and in Theorems 4, 5 we will see that all the results of the Sections 4 and 5 are included in the scattering relations for the problem $\left(P_{\Lambda}\right)$, provided that $\Lambda$ is self-adjoint. In particular we have the following.

COROLLARY 4. If $\Lambda$ is a self-adjoint linear operator, then the normalized scattering amplitude of the problem $\left(P_{\Lambda}\right)$ satisfies the classical reciprocity relation, basic scattering theorem and the optical theorem. 
Furthermore, in view of (87) the relation (85) takes the form (56). Hence, for the operator $G$ the following holds.

COROLLARY 5. If $\Lambda$ is a self-adjoint linear operator, then the scattering amplitude operator $G$ corresponding to the problem $\left(P_{\Lambda}\right)$ has a countable number of eigenvalues. All the eigenvalues $\lambda \in \mathbb{C}$ lie on the circle $|\lambda|^{2}+4 \pi \operatorname{Re} \lambda=0$.

\section{Acknowledgement}

The author thanks the referee for his comments and suggestions, that led to the present version of the paper.

\section{References}

[1] T. S. Angell, R. E. Kleinman and F. Hettlich, "The resistive and conductive problems for the exterior Helmholtz equation", SIAM J. Appl. Math. 50 (1990) 1607-1623.

[2] C. Athanasiadis, "Low-frequency electromagnetic scattering theory for a multi-layered scatterer", Q. J. Mech. Appl. Math. 44 (1991) 55-67.

[3] C. Athanasiadis, "The hard-core multi-layered ellipsoid in a low-frequency acoustic field", Int. J. Eng. 32 (1994) 1352-1359.

[4] C. Athanasiadis, "The multi-layered ellipsoid with a soft core in the presence of a low-frequency acoustic wave", Q. J. Mech. Appl. Math. 47 (1994) 441-459.

[5] C. Athanasiadis and I. G. Stratis, "Low-frequency acoustic scattering by a infinitely stratified scatterer", Rend. Mat. 15 (1995) 133-152.

[6] D. Colton and R. Kress, Integral equation methods in Scattering theory, (John Wiley, New York, 1983).

[7] D. Colton and R. Kress, Inverse acoustic and electromagnetic scattering theory (Springer, New York, 1992).

[8] D. Colton and R. Kress, "Eigenvalues of the far field operator and inverse scattering theory", SIAM J. Appl. Math. 26 (1995) 601-615.

[9] G. Dassios, "Second order low-frequency scattering by the soft ellipsoid", SIAM J. Appl. Math. 38 (1980) 373-381.

[10] G. Dassios, "Low-frequency scattering theory for a penetrable body with an impenetrable core", SIAM J. Appl. Math. 42 (1982) 272-280.

[11] G. Dassios, K. Kiriaki and D. Polysos, "On the scattering amplitudes for ealstic waves", J. Appl. Math. Phys. 38 (1987) 856-873.

[12] B. DeFacio, "Classical, linear, electromagnetic impedance theory with infinite integrable discontinuites", J. Math. Phys. 31 (1990) 2155-2164.

[13] D. S. Jones, Acoustic and electromagnetic scattering (Clarendon Press, Oxford, 1986).

[14] R. E. Kleinman and P. A. Martin, "On single integral euqations for the transmission problem of acoustics", SIAM J. Appl. Math. 44 (1988) 307-325.

[15] R. E. Kleinman and T. B. A. Senior, In low and high frequency asymptotics, Volume 2 (eds. V. K. Varadan and V. V. Varadan, Elsevier, Amsterdam, 1986) 1-70. 
[16] R. Kress and G. F. Roach, "Transmission problems for the Helmholtz equation", J. Math. Phys. 19 (1975) 1433-1437.

[17] C. Miranda, Partial differential equations of elliptic type (Springer, New York, 1970).

[18] P. C. Sabatier, "On modelling discontinuous media. Three dimensional scattering", J. Math. Phys. 30 (1989) 2585-2598.

[19] V. Twersky, "Certain transmissions and reftection theorems", J. Appl. Phys. 25 (1954) 859-862.

[20] V. Twersky, "Multiple scattering of electromagnetic waves by arbitrary configurations", J. Math. Phys. 8 (1966) 589-598. 